\title{
Sedación, Seguridad y Utilidad de la Endoscopia Digestiva Alta en Pediatría
}

\author{
FRANCISCA RIERA C. ${ }^{1}$, CARMEN GLORIA GONZÁLEZ F. ${ }^{2}$, \\ CARINA VACA Z. ${ }^{1}$, PAMELA MARCHANT A. ${ }^{3}$, CONSTANZA GLENZ A. ${ }^{1}$, \\ FRANCISCO LARRAÍN B. ${ }^{3}$, PAUL HARRIS R. ${ }^{3}$ \\ 1. Residentes de Pediatría. Facultad de Medicina, Pontificia Universidad Católica de Chile. \\ 2. Interna de Medicina. Facultad de Medicina, Pontificia Universidad Católica de Chile. \\ 3. Pediatra Gastroenterólogo, Departamento de Pediatría, Unidad de Gastroenterología y Nutrición, Facultad de Medicina, \\ Pontificia Universidad Católica de Chile.
}

\begin{abstract}
Sedation, Safety and Usefulness of Upper Gastrointestinal Endoscopy in Pediatrics

Introduction: Upper gastrointestinal studies are routine diagnostic and therapeutic procedures. In pediatrics, however, they are limited by the need for sedation and monitoring. Objectives: To evaluate sedation, indication, and parent perception of these exams. Patients and Methods: A prospective study of 190 pediatric upper gastrointestinal endoscopies was undertaken. Demographic data, as well as information regarding the exam was obtained. Results: Patients' average age was $8.5 \pm 4.2$, mostly referred by pediatricians $(60 \%)$ Main reason for referral was recurrent abdominal pain $(29.8 \%)$. Patients were classified according to ASA criteria ( $93 \%$ ASA I and II). Most patients required two or more medications for optimal sedation, with satisfactory procedure in over $90 \%$. Over $75.5 \%$ had complete amnesia, $42.7 \%$ presented minor discomfort after the procedure. Findings of these procedures included $61 \%$ of normal exams, $13.9 \%$ esophagitis. More findings were there result of analyses according to children's weight (over or under $14 \mathrm{~kg}$ ), age, ASA, reason for referral, nurse's evaluation of sedation. Conclusion: Ambulatory endoscopic procedures can be performed safely in children, with moderate sedation. Requirements are adequate monitoring, and deep knowledge of resuscitation techniques.

(Key words: Children, gastrointestinal endoscopy, sedation).

Rev Chil Pediatr 2010; 81 (1): 37-45
\end{abstract}

\section{RESUMEN}

Introducción: El estudio endoscópico alto es un procedimiento diagnóstico y terapéutico considerado de rutina, pero su realización en pediatría se encuentra limitada dada la necesidad de sedación y monitoreo adecuados. Objetivos: Evaluar la sedación, indicación y percepción de los padres del examen. Materiales y Métodos: Estudio prospectivo de 190 niños sometidos a endoscopia digestiva alta. Se obtuvieron datos biodemográficos, tipo de sedación y resultados del examen. Resultados: La edad fue 8,5+4,2 años, la mayoría

Trabajo recibido el 06 de marzo de 2009, devuelto para corregir el 06 de abril de 2009, segunda versión el 22 de septiembre de 2009, tercera versión el 09 de diciembre de 2009, aceptado para publicación el 14 de diciembre de 2009.

Correspondencia a:

Dr. Paul R Harris

E-mail: pharris@med.puc.cl 
referidos por pediatra $(60 \%)$. La principal indicación del estudio fue el dolor abdominal recurrente $(29,8 \%)$. Los pacientes fueron clasificados de acuerdo al ASA (93\% ASA I y II). En la mayoría se requirió de 2 ó más drogas para lograr sedación óptima. La calidad del procedimiento fue satisfactoria en más del $90 \%$. Un 75,5\% presentaron amnesia completa y un $42,7 \%$ molestias leves posterior al procedimiento. Entre los hallazgos destacaron signos de esofagitis en el $13,9 \%$, observándose un $61 \%$ de los exámenes normales. Al analizar los pacientes por peso (mayores y menores de $14 \mathrm{~kg}$ ), se encontraron diferencias en edad ( $<<0,00001$ ), ASA ( $p$ $=0,001)$, indicación de endoscopia $(\mathrm{p}=0,001)$ y evaluación de la sedación por enfermera $(\mathrm{p}=0,038)$. Conclusión: Procedimientos endoscópicos en niños pueden realizarse de manera segura en forma ambulatoria mediante sedación moderada. Una adecuada monitorización y conocimiento de técnicas de reanimación son básicos al momento de realizar dichos procedimientos.

(Palabras clave: Niños, endoscopia digestiva, sedación).

Rev Chil Pediatr 2010; 81 (1): 37-45

\section{Introducción}

El estudio endoscópico alto (EDA) es un procedimiento diagnóstico y terapéutico considerado de rutina en la práctica gastroenterológica diaria. Sin embargo, su realización en pediatría se encuentra limitada a pocos centros, dada la necesidad de sedación y monitoreo adecuados, especialmente en lactantes menores, en quienes muchas veces se requiere de hospitalización y anestesia general para efectuar dicho procedimiento ${ }^{1-4}$.

Dada la amplia variación en la práctica clínica diaria y metódica de la EDA en niños y sus técnicas de sedación en el medio local ${ }^{5,6}$, las que pueden incluir una sedación moderada (técnica en la que el paciente puede ser despertado fácilmente y es capaz de mantener sus reflejos protectores y permeabilidad de vía aérea), sedación profunda (técnica por el cual el paciente no puede ser despertado fácilmente, que puede o no acompañarse de la pérdida de reflejos protectores y permeabilidad de vía aérea), y anestesia general (estado en el cual el paciente no siente dolor ni puede ser despertado), sumado a la falta de datos nacionales sobre la factibilidad de realizar en forma exitosa y segura este procedimiento mediante sedación moderada, se diseñó este estudio prospectivo cuyos 4 objetivos fueron evaluar: i) En sedación moderada, tipo y número de drogas utilizadas (benzodiacepinas, barbitúricos, ketamina, opioides, etc) y necesidad de uso de antagonistas específicos; ii) indicación de la EDA (diagnóstica o terapéutica), instrumentos utilizados, duración del procedimiento; iii) percepción subjetiva de la calidad del examen, y iv) considerando la dificultades técnicas del uso de instrumentos endoscópicos en lactantes menores, se estudiaron los requerimientos de sedación, monitoreo y realización del procedimiento en lactantes comparados con niños mayores.

\section{Pacientes y Método}

\section{Pacientes}

Se enrolaron 190 niños en forma consecutiva, prospectiva y protocolizada, referidos a la Unidad de Endoscopia del Hospital Clínico de la Pontificia Universidad Católica de Chile, previo consentimiento de sus padres. Los criterios de inclusión fueron las indicaciones de endoscopia de los médicos tratantes de los niños, quienes solicitaron el examen y se consideraron criterios de exclusión: pacientes mayores de 18 años, los presentaban alguna patología grave asociada y aquellos niños que no tenían un teléfono de contacto.

\section{Datos Biodemográficos}

Se registró nombre, edad, sexo, peso, médico referente, situación (hospitalizado o ambulatorio), e indicación del procedimiento según lo establecido en la orden de examen.

\section{Preparación y Monitoreo del Procedimiento}

Se solicitó un tiempo de ayuno mínimo de 6 horas. Los procedimientos se realizaron en la Unidad de Endoscopia si el paciente pesaba 
más o igual a $14 \mathrm{~kg}$. En dicha sala se cuenta con un monitor de saturación y de frecuencia cardíaca. La sedación fue administrada por el endoscopista a cargo del procedimiento. Cuando el niño pesaba menos de $14 \mathrm{~kg}$, el procedimiento era realizado en una sala de procedimientos supervisado por un intensivista pediátrico, quien indicaba además la sedación. Dicha sala cuenta con monitor cardiorrespiratorio, saturómetro, capnógrafo y equipo de reanimación avanzada.

\section{Sedación}

Cada paciente fue individualizado de acuerdo a la clasificación de riesgo anestésico internacional ASA, se hizo un registro de las drogas utilizadas, dosis, vía de administración, efectos adversos y uso de antagonistas específicos. Cada paciente permaneció en observación en una sala destinada a ello por lo menos $30 \mathrm{mi}-$ nutos cuando el procedimiento era ambulatorio, o bien fue trasladado a su habitación cuando el procedimiento se efectuó con el paciente hospitalizado.

\section{Procedimiento}

Se registraron los siguientes datos: instrumento utilizado, duración, diagnóstico macroscópico, presencia en la sala de los padres, requerimiento de apoyo de enfermera y la calidad del procedimiento catalogándolo como satisfactorio o insatisfactorio. Adicionalmente se le pidió al endoscopista, intensivista y enfermera, que catalogaran la sedación como: muy buena, buena, regular o mala.

\section{Seguimiento}

Al día siguiente del procedimiento, se realizó una entrevista telefónica a los padres o al niño cuando éste era mayor de 10 años, inquiriendo sobre duración de los efectos de la sedación, presencia de la amnesia, tolerancia a la alimentación y presencia de efectos adversos. En niños hospitalizados, el seguimiento se realizó personalmente. Los padres respondieron la entrevista en los niños menores de 10 años.

\section{Análisis Estadístico}

Se utilizó una ficha protocolizada para recolectar los datos, los resultados que fueron analizados con el programa estadístico SPSS. $\mathrm{El}$ análisis estadístico fue realizado mediante $\mathrm{t}$ de Student para muestras independientes para los datos numéricos y con $\chi^{2}$ de Pearson para datos categóricos. Se consideró como significativo un valor $\mathrm{p}<0,05$.

\section{Resultados}

Se incluyó a 190 pacientes de los cuales $23(12,1 \%)$ eran menores de $14 \mathrm{~kg}$. La edad promedio fue de 8,5 + 4,2 años (x + 1DS), con un peso promedio de $32+15,1 \mathrm{~kg}$. Los pacientes fueron clasificados de acuerdo a la clasificación internacional ASA. E1 82,1\% de los pacientes se catalogó como ASA I (tabla 1). La procedencia de los pacientes fue en su mayoría del policlínico institucional $(60,5 \%)$ y pensionado de pediatría $(16,8 \%)$ (tabla 2$)$. Dos tercios de los pacientes fueron referidos por un pediatra, el resto por otros especialistas (tabla 2). Las indicaciones del estudio difirieron según la edad. En el grupo de niños mayores la principal indicación fue el dolor abdominal recurrente (DAR) con sospecha de organicidad $(33,1 \%)$ y la patología esofágica $(31,9 \%)$, en cambio, en los lactantes fue la sospecha de enfermedad celíaca $(27,3 \%)(p=0,001)$ (tabla 3$)$.

En el $90 \%$ de los pacientes cuyo peso era superior o igual a $14 \mathrm{~kg}$, el examen se realizó en forma ambulatoria, comparados con un 62 $\%$ en los niños cuyo peso era inferior. El período de ayuno promedio fue de $9 \pm 4$ hrs y $11 \pm 2$ hrs, respectivamente $(\mathrm{p}=\mathrm{NS})$ (tabla 4$)$.

El instrumento utilizado varió de acuerdo a la edad del niño e incluyó endoscopios de fibra y videoendoscopios. No obstante, el 39,3\% de los niños fue evaluado con un panendoscopio de fibra de 7,9 mm de diámetro (Olympus GIFXP20) y el 37,9\% con un video panendoscopio de 9,8 mm (Olympus GIF-130). La duración del examen fue de $12 \pm 6$ minutos (tabla 4). En el 8,4\% de los procedimientos realizados en los niños de más o igual a $14 \mathrm{~kg}$ de peso, los padres estuvieron presentes, lo cual fue decidido por el endoscopista quien ejecutó el procedimiento y que generalmente fue reflejo del grado de ansiedad de los mismos. En el 88\% de los procedimientos hubo presencia directa 
de una enfermera y de apoyo paramédico. No se encontró diferencia significativa entre los grupos mayores y menores con respecto a la presencia de los padres, apoyo de personal ni duración del procedimiento (tabla 4).
Las drogas más usadas durante el procedimiento fueron Midazolam y Meperidina. Midazolam fue usado en el 99,1\% de los pacientes, a dosis promedio de $0,13 \mathrm{mg} / \mathrm{kg}$. La segunda droga utilizada más utilizada fue la

\section{Tabla 1. Características demográficas}

\begin{tabular}{|c|c|c|c|c|}
\hline Características & $\begin{array}{c}\text { Niños }<14 \mathrm{~kg} \\
n=23\end{array}$ & $\begin{array}{c}\text { Niños } \geq 14 \mathrm{~kg} \\
n=167\end{array}$ & $\begin{array}{c}\text { Total } \\
n=190\end{array}$ & Valor $\mathbf{p}^{*}$ \\
\hline $\begin{array}{l}\text { Edad en años, } x \pm 1 \mathrm{DE} \\
\text { (rango) }\end{array}$ & $\begin{array}{l}1,5 \pm 0,7 \\
(0,4-2,9)\end{array}$ & $\begin{array}{l}9,5 \pm 3,5 \\
(3-17)\end{array}$ & $\begin{array}{l}8,5 \pm 4,2 \\
(0,4-17)\end{array}$ & $<0,00001$ \\
\hline $\begin{array}{l}\text { Peso en } \mathrm{kg} x \pm 1 \mathrm{DE} \\
\text { (rango) }\end{array}$ & $\begin{array}{l}10 \pm 2 \\
(6-13)\end{array}$ & $\begin{array}{r}35 \pm 13 \\
(14-72)\end{array}$ & $\begin{array}{c}32 \pm 15,1 \\
(6-72)\end{array}$ & $<0,00001$ \\
\hline \multicolumn{5}{|l|}{ Sexo N $\left.{ }^{\circ} \%\right)$} \\
\hline Femenino & $9(39,1)$ & $74(44,3)$ & $83(43,6)$ & NS \\
\hline Masculino & $14(60,9)$ & $93(55,7)$ & $107(54,4)$ & \\
\hline \multicolumn{5}{|l|}{ ASA No (\%) } \\
\hline I & $14(60,9)$ & $142(85)$ & $156(82,1)$ & 0,001 \\
\hline ॥ & $3(13)$ & $18(10,8)$ & $21(11)$ & \\
\hline III & $5(21,7)$ & $7(4,2)$ & $12(6,3)$ & \\
\hline IV & $1(4,3)$ & $0(0)$ & $1(0,5)$ & \\
\hline
\end{tabular}

*Se realizó t de Student para muestras independientes para edad y peso. Se realizó $\chi^{2}$ para comparación de sexo y ASA.

Tabla 2. Procedencia de pacientes y especialista que deriva

\begin{tabular}{|c|c|c|c|}
\hline & Niños < 14 kg & Niños $\geq 14$ kg & Total \\
\hline \multicolumn{4}{|l|}{ Procedencia*, № (\%) } \\
\hline Policlínico institucional & $2 \quad(8,7)$ & $113(67,7)$ & $115(60,5)$ \\
\hline Pensionado de pediatría & $7 \quad(30,4)$ & $25(15)$ & $32(16,8)$ \\
\hline Consulta privada & $7 \quad(30,4)$ & $11 \quad(6,6)$ & $18 \quad(9,5)$ \\
\hline Servicio de pediatría & $5 \quad(21,7)$ & $5 \quad(3)$ & $(5,3)$ \\
\hline Servicio de urgencia & $1 \quad(4,3)$ & $8 \quad(4,8)$ & $(4,7)$ \\
\hline Otros & $0 \quad(0)$ & $5 \quad(3,0)$ & $5 \quad(2,7)$ \\
\hline UCl & $1 \quad(4,3)$ & $0 \quad(0)$ & $1 \quad(0,5)$ \\
\hline Total & $23(100)$ & $167(100)$ & $190(100)$ \\
\hline \multicolumn{4}{|l|}{ Referente $^{*}, \mathrm{~N}^{\circ}(\%)$} \\
\hline Pediatra & $1 \quad(4,3)$ & $107 \quad(68,2)$ & $108(60)$ \\
\hline Cirujano infantil & $1 \quad(4,3)$ & $36 \quad(22,9)$ & $37(20,6)$ \\
\hline Gastroenterólogo & $19(82,6)$ & $13 \quad(8,3)$ & $32(17,8)$ \\
\hline Otros & $2 \quad(8,7)$ & $10 \quad(5,9)$ & $12 \quad(6,3)$ \\
\hline Total & $23(100)$ & $167(100)$ & $190(100)$ \\
\hline
\end{tabular}

* valor $\mathrm{p}=$ NS por $\chi^{2}$. 
Meperidina (59\%) a una dosis promedio de $0,82 \mathrm{mg} / \mathrm{kg}$. En la mayoría de los niños se requirió de 2 ó más drogas para lograr un nivel óptimo de sedación. Sólo 2 niños requirieron uso de antagonistas: uno requirió naloxona y el otro flumazenil. Ambos respondieron rápidamente y sin incidentes. De los 23 ni- ños menores de $14 \mathrm{~kg}, 20$ recibieron oxígeno durante el procedimiento, 19 por bigotera y en un caso debió utilizarse mascarilla. En el grupo de niños menores, 10 de ellos (34\%) presentaron desaturación, que alcanzó como máximo 69\%. En el grupo de niños mayores se utilizó apoyo con oxígeno en el 20\% de los

Tabla 3. Indicaciones del estudio endoscópico

\begin{tabular}{|c|c|c|c|}
\hline Indicación, $\mathbf{N}^{\circ}(\%)$ & Niños $<14$ kg & Niños $\geq 14$ kg & Total*** \\
\hline 1. Patología esofágica & $4(13,6)$ & $53 \quad(31,9)$ & $57 \quad(30,3)$ \\
\hline 2. $\mathrm{DAR}^{*}+$ sospecha de organicidad & $1 \quad(4,5)$ & $55 \quad(33,1)$ & $56(29,8)$ \\
\hline 3. Sospecha enfermedad celíaca & $6(27,3)$ & $14 \quad(8,4)$ & $20(10,6)$ \\
\hline 4. Vómitos crónicos & $3(13,6)$ & $(6,6)$ & $(7,4)$ \\
\hline 5. Hemorragia digestiva alta & $3(13,6)$ & $(6,6)$ & $(7,4)$ \\
\hline 6. DAR funcional & $0 \quad(0)$ & $(5,4)$ & $(4,8)$ \\
\hline 7. Cuerpo extraño & $4(13,6)$ & $(2,4)$ & $(3,2)$ \\
\hline 8. Gastrostomía & $2 \quad(9,1)$ & $(2,4)$ & $(3,2)$ \\
\hline 9. Sospecha recidiva UP** & $0 \quad(0)$ & (3) & $(2,7)$ \\
\hline 10. $\mathrm{DAR}^{*}+$ antecedente familiar UP** & $0 \quad(0)$ & $1 \quad(0,6)$ & $(0,5)$ \\
\hline Total & $23(100)$ & $167(100)$ & $190(100)$ \\
\hline
\end{tabular}

*DAR: dolor abdominal recurrente. ${ }^{* *}$ UP: úlcera péptica. ${ }^{* * *} p=0,001$ por $\chi^{2}$.

Tabla 4. Características del procedimiento

\begin{tabular}{|c|c|c|c|c|}
\hline & & Niños $<14$ kg & Niños $\geq 14$ kg & Total \\
\hline Duración de ayuno horas*, $X \pm 1 \mathrm{DE}$ & & $9 \pm 4$ & $11 \pm 2$ & $11 \pm 2$ \\
\hline $\begin{array}{l}\text { Duración del procedimiento } \\
\text { Minutos* }, X \pm 1 D E\end{array}$ & & $11 \pm 7$ & $12 \pm 6$ & $12 \pm 6$ \\
\hline \multirow{2}{*}{$\begin{array}{l}\text { Presencia de padres, } \\
\mathrm{N}^{\circ}(\%)\end{array}$} & No & $23(100)$ & $153(91,6)$ & $175(92,6)$ \\
\hline & Sí & $0 \quad(0)$ & $14(8,4)$ & $14(7,4)$ \\
\hline \multirow{2}{*}{$\begin{array}{l}\text { Apoyo de enfermera, } \\
\mathrm{N}^{\circ}(\%)\end{array}$} & No & $0 \quad(0)$ & $21(12,6)$ & $21(11,1)$ \\
\hline & Sí & $23(100)$ & $146(87,4)$ & $168(88,9)$ \\
\hline \multirow{2}{*}{$\begin{array}{l}\text { Apoyo paramédico, } \\
\mathrm{N}^{\circ}(\%)\end{array}$} & No & $2(10)$ & $4(2,4)$ & $6(3,2)$ \\
\hline & Sí & $22(90)$ & $161(97,6)$ & $179(96,8)$ \\
\hline Personas presentes*, $\mathrm{X} \pm 1 \mathrm{DE}$ & & $5 \pm 1$ & $4 \pm 1$ & $4 \pm 1$ \\
\hline Horas dormido*, $X \pm 1 \mathrm{DE}$ & & $2 \pm 3$ & $1 \pm 1$ & $1 \pm 1$ \\
\hline \multirow{3}{*}{$\begin{array}{l}\text { Presencia de amnesia**, } \\
\text { № (\%) }\end{array}$} & No & $0 \quad(0)$ & $5(3,3)$ & $5(3,1)$ \\
\hline & Sí & $9(90)$ & $114(74,5)$ & $123(75,5)$ \\
\hline & Parcial & $1(10)$ & $34(22,2)$ & $35(21,5)$ \\
\hline
\end{tabular}

* $p=$ NS para las variables indicadas. La presencia de padres, enfermera y paramédicos son diferentes por construcción, ya que es parte del procedimiento en el grupo de niños menores. ${ }^{* *}$ Los datos faltantes para completar los totales de niños, corresponden a entrevistas en que la respuesta fue "no se o no me acuerdo". 
casos, aún cuando sólo en el $8 \%$ de los niños se documentó desaturación, la que alcanzó un máximo del 75\%.

De los resultados macroscópicos de la EDA destaca la presencia de signos de esofagitis en el $13,9 \%$ de los pacientes, un $61 \%$ de los exámenes fueron considerados normales. No se encontraron diferencias estadísticas en los hallazgos al separarlos por peso (tabla 5).

Respecto de la calidad de la endoscopia, sedación y hallazgos, en el grupo de niños cuyo peso era inferior a $14 \mathrm{~kg}$, el $86 \%$ de los procedimientos fueron considerados muy buenos o buenos por el endoscopista. Esta cifra aumen- tó al 90\% cuando la pregunta fue hecha al intensivista y a un $100 \%$, cuando fue hecha al personal de apoyo (figura 1). Estos porcentajes bajan al considerar al grupo de niños de más de $14 \mathrm{~kg}$ de peso, sólo siendo la diferencia significativa entre ambos grupos cuando se le preguntó al personal de enfermería $(\mathrm{p}=0,038)$.

Los resultados del seguimiento telefónico, muestran que un 75,5\% de los niños presentaron amnesia completa (tabla 4 ) y un 57,3\% no presentó ninguna molestia posterior al procedimiento (tabla 6). La tolerancia a la primera alimentación fue reportada como buena en el $97,4 \%$ de los niños.

Niños peso $<14$ kg

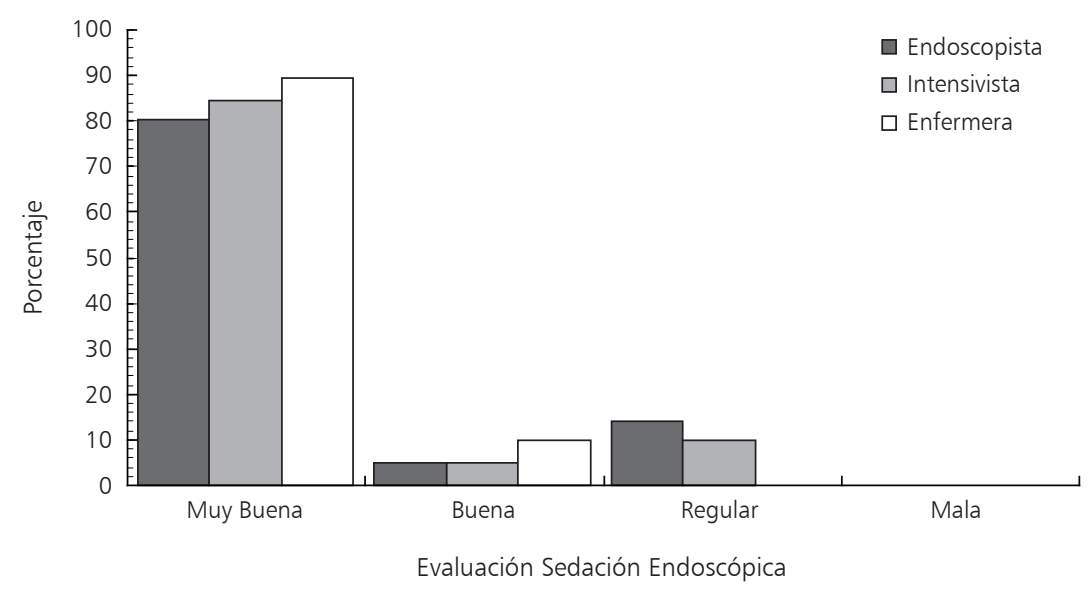

Niños peso $\geq 14 \mathrm{~kg}$

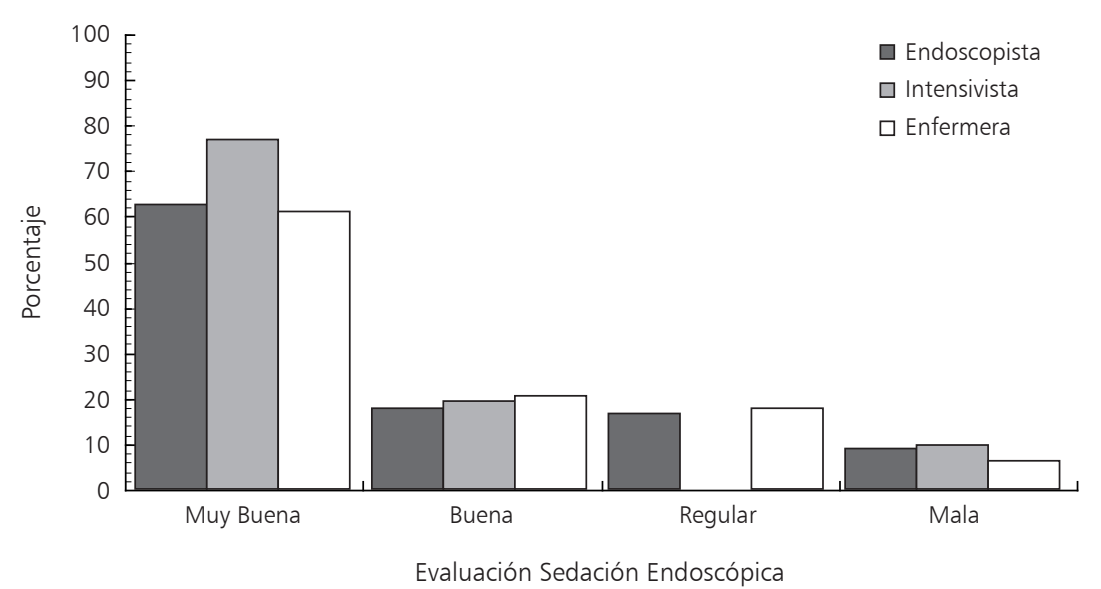

Figura 1. Evaluación de la sedación en niños $<14 \mathrm{~kg}$ (panel superior) $\mathrm{y}>14 \mathrm{~kg}$ (panel inferior) por endoscopista, intensivista $\mathrm{y}$ enfermera. El intensivista participa en la evaluación de lactantes y de niños menores de $14 \mathrm{~kg}$. No hay diferencia en la evaluación de ambos grupos por intensivista y endoscopista. $\mathrm{p}=0,038$ por $\chi^{2}$ para evaluación por enfermera. 
Tabla 5. Hallazgos macroscópicos en endoscopia

\begin{tabular}{|c|c|c|c|}
\hline Hallazgos*, $\mathrm{N}^{\circ}(\%)$ & Niños $<14$ kg & Niños $\geq 14 \mathrm{Kg}$ & Total** \\
\hline Normal & $13(59,1)$ & $101(61,2)$ & $114(61)$ \\
\hline Esofagitis & $1 \quad(4,5)$ & $25 \quad(15,2)$ & $26 \quad(13,9)$ \\
\hline Gastropatía & $1 \quad(4,5)$ & $18(10,9)$ & $20 \quad(10,7)$ \\
\hline Nodularidad antral & $1(4,5)$ & $7 \quad(4,2)$ & $8 \quad(4,3)$ \\
\hline Patología duodenal no UP*** & $3(13,6)$ & $2 \quad(1,2)$ & $(2,7)$ \\
\hline UP* activa/cicatrizada & $1 \quad(4,5)$ & $3 \quad(1,8)$ & $(2,1)$ \\
\hline Várices esofágicas & $0 \quad(0)$ & $4 \quad(2,4)$ & $(2,1)$ \\
\hline Otro & $0 \quad(0)$ & $(2,4)$ & $(2,1)$ \\
\hline Cuerpo extraño & $2 \quad(9,1)$ & $1 \quad(0,6)$ & $(1,1)$ \\
\hline Total & $22(100)$ & $165(100)$ & $187(100)$ \\
\hline
\end{tabular}

* $\mathrm{p}=$ NS. ${ }^{* *}$ Hallazgos macroscópicos de 187 pacientes estudiados, 3 de ellos sin datos. ***UP: úlcera péptica.

Tabla 6. Efectos adversos de la sedación

\begin{tabular}{|c|c|c|c|}
\hline Efectos Adversos (\%)* & Niños $<14$ kg & Niños $\geq 14$ kg & Total** \\
\hline No refiere & $16(68,4)$ & $93(55,6)$ & $109(57,3)$ \\
\hline Odinofagia & $1 \quad(5,3)$ & $38(22,6)$ & $39(20,3)$ \\
\hline Dolor abdominal & $1 \quad(5,3)$ & $10 \quad(5,6)$ & $(5,6)$ \\
\hline Vómitos & $1 \quad(5,3)$ & $10 \quad(5,6)$ & $(5,6)$ \\
\hline Otros & $0 \quad(0)$ & $(5,6)$ & $(4,9)$ \\
\hline Irritabilidad & $4(15,8)$ & $(0,8)$ & $(2,8)$ \\
\hline Náuseas & $0 \quad(0)$ & $(1,6)$ & $(1,4)$ \\
\hline Decaimiento & $0 \quad(0)$ & $(0,8)$ & $(0,7)$ \\
\hline Mareos & $0 \quad(0)$ & $1 \quad(0,8)$ & $1 \quad(0,7)$ \\
\hline Eritema sitio punción & $0 \quad(0)$ & $1 \quad(0,8)$ & $1 \quad(0,7)$ \\
\hline Total & $23(100)$ & $167(100)$ & $190(100)$ \\
\hline
\end{tabular}

* \% calculado sobre respuesta recibidas. ${ }^{* *} p=$ NS.

\section{Discusión}

La realización de la EDA en pediatría requiere de más tiempo, paciencia y experiencia en el manejo de los factores emocionales del niño y de la familia ${ }^{1,2}$. Esto debido a que la mayoría de las complicaciones derivadas de estos procedimientos son secundarias a la necesidad de lograr un grado óptimo de sedación, la que permita no sólo efectuar el examen sino también manejar la ansiedad y molestias derivadas de éste. A partir de esta necesidad, los niños pueden requerir desde una sedación moderada hasta la anestesia general. Para decidir el tipo de sedación a utilizar, se deben analizar factores tales como la edad, diagnóstico, condición clínica, nivel de ansiedad, experiencia y preferencias del operador y los recursos con los que cuenta el centro médico. Sin embargo, es la correcta elección del paciente la medida de seguridad más importante en cualquier protocolo de sedación ${ }^{7}$.

Existe gran variabilidad en las técnicas de sedación utilizadas en los procedimientos gastroenterológicos. En un estudio realizado por Lightdale y cols, encuestaron a 103 gastroen- 
terólogos pediatras sobre las técnicas de sedación utilizadas en sus pacientes, se observó que el $10 \%$ prefería utilizar anestesia general, mientras que el $23 \%$ sólo utilizaba sedación endovenosa, el resto de los encuestados utilizaba ambas técnicas. Sin embargo, independiente de la técnica utilizada, el $77 \%$ suplementaba con oxígeno al paciente ${ }^{4}$. Es evidente de los resultados que los niños menores exhiben mayor riesgo general expresado en las diferencias encontradas en la clasificación ASA. No obstante, no encontramos ninguna diferencia en aspectos relacionados al uso de endoscopios de distinto diámetro (datos no mostrados), reflejando probablemente elección personal del endoscopista, experiencia y disponibilidad.

Los fármacos utilizados con mayor frecuencia para la sedación son benzodiazepinas, opiodes y ketamina, los que generalmente deben combinase para lograr el nivel de sedación deseada $^{4,8}$. Recientemente se ha descrito con éxito el uso de propofol en diferentes procedimientos $^{9-11}$. En un estudio prospectivo, Lightdale y cols, evaluaron el uso de propofol como agente para sedación en procedimientos endoscópicos en niños, sin embargo, al compararlo con agentes de uso común como midazolam y fentanilo, no observaron mayores beneficios ${ }^{11}$.

Las complicaciones derivadas de estos procedimientos difieren de las descritas en adultos, debido a las técnicas de sedación y las diferentes patologías asociadas. Thakkar y cols, estudiaron retrospectivamente 10234 procedimientos endoscópicos altos en niños, el $46 \%$ se realizaron con sedación endovenosa, el resto bajo anestesia general; $2,3 \%$ de los pacientes presentó alguna complicación, siendo la más frecuente la hipoxia. Se determinó que los pacientes de menor edad, sexo femenino, mayor ASA y aquellos con sedación endovenosa presentan mayor riesgo de complicaciones $(\mathrm{p}<0,001)$. De esto se concluye que el uso de estos medicamentos debe realizarse con extremo cuidado debido al riesgo de hipoxia, la que ocurrió en el 34\% de los niños menores y $8 \%$ de los mayores. Es por tanto imperioso que los profesionales quienes lleven a cabo estos procedimientos, tengan conocimientos en técnicas de monitorización y de reanimación pediátrica $^{12}$.
En nuestra serie la mayoría de los pacientes fueron ambulatorios, derivados por pediatras para estudio de DAR. Un estudio nacional llevado a cabo por Daza y cols, evaluaron retrospectivamente las indicaciones y resultados en 926 EDA realizadas a niños de entre 1 mes a 15 años. La principal indicación del estudio fue el DAR (20,8\%). Sin embargo, la mayoría de los niños tuvieron una EDA normal ${ }^{5}$; estos resultados son concordantes con los descritos por nuestro grupo. A partir de esto se desprende que si bien, los criterios para derivar a un niño a un estudio endoscópico son más estrictos que en adultos, no es infrecuente la ausencia de signos orgánicos que expliquen la sintomatología.

Los procedimientos fueron considerados satisfactorios en más del $90 \%$ de los pacientes y un $75,5 \%$ de ellos repostaron (sus padres o el paciente) amnesia completa, con pocos y leves efectos secundarios. Sin embargo, el tiempo de sueño después del procedimiento fue mayor al destinado para la observación, esto refuerza la importancia de utilizar medicamentos de vida media corta y de una monitorización rigurosa posterior al procedimiento. Existen diferentes maneras de evaluar el mejor momento para determinar el alta, algunas de ellas engorrosas y con tendencia a subestimar la profundidad de la sedación. A partir de esto, Malviya y cols, estudiaron prospectivamente a 29 niños sedados para estudio ecocardiográfico. Concluyeron que si el niño es capaz de mantenerse despierto por más de 20 minutos en un ambiente tranquilo, puede ser dado de alta con tranquili$\mathrm{dad}^{13}$. La enfermera es la profesional que mas tiempo pasa con el paciente, al recibirlo, participar en el procedimiento, y supervisar el alta al finalizar el período de observación, lo que podría explicar las diferencias reportadas en la apreciación de la calidad de la sedación.

Los resultados obtenidos en este estudio son consistentes con los descritos previamente por nuestro grupo en endoscopias bajas. En aquella oportunidad se evaluó de forma prospectiva la seguridad y tolerancia a la colonoscopia en 123 niños, concluyéndose que este procedimiento puede ser llegado a cabo con sedación moderada y efectos adversos mínimos ${ }^{14}$.

E1 2006 la Academia Americana de Pedia- 
tría definió las metas de sedación en niños, estas incluyen la seguridad, minimizar el dolor, controlar la ansiedad, maximizar la amnesia, controlar la conducta del niño para poder realizar el procedimiento y supervisarlo hasta que pueda ser dado de alta de forma segura ${ }^{3}$. Es por tanto de vital importancia el juicio clínico, para reconocer las diferencias psicológicas y fisiológicas de los niños, teniendo en cuenta de que el propósito al realizar un procedimiento en pediatría no puede ser solamente diagnóstico ni terapéutico. Nuestro estudio demuestra que estas metas son alcanzables y pueden ser cumplidas en un número importante de pacientes, sin diferencias importantes entre lactantes y niños mayores, definidos en este estudio como mayores y menores de $14 \mathrm{~kg}$, respectivamente.

\section{Conclusiones}

En resumen, los procedimientos endoscópicos altos pueden ser realizados de manera segura con una técnica de sedación moderada, tanto en pacientes ambulatorios como hospitalizados, incluidos lactantes. Los lactantes menores tiene mas riesgo, pero no son diferentes en sexo, patología o medico referente que los niños mayores, aun cuando hay diferencias en los motivos de la endoscopia reflejando las patologías prevalentes. Sin embargo, una adecuada monitorización y conocimiento de técnicas de reanimación son básicos al momento de realizar dichos procedimientos, y permite encontrar similitudes en la duración del procedimiento, duración de la recuperación, y en la presencia efectos adversos. Es necesario realizar un mayor número de estudios con el fin de estandarizar las diferentes técnicas de sedación y monitorización para ofrecer a los pacientes una mayor seguridad y eficacia.

\section{Referencias}

1.- Tolia V, Peters J, Gilger M: Sedation for pediatric endoscopic procedures. J Pediatr Gastroenterol Nutr 2000; 30: 477-85.
2.- Lee K, Anderson M, Baron T, Banerjee S, Cash BD, Dominitz JA, et al: Modification in endoscopic practice for pediatric patients. Gastrointest Endosc 2008; 1: 1-9.

3.- Cote CJ, Wilson S, and the Work Group on Sedation: Guidelines for monitoring and managment of pediatric patients during and after sedation for diagnostic and therapeutic procedures: an update. Pediatrics 2006; 118: 2587-602.

4.- Lightdale J, Mahoney L, Schwarz S, Liacouras C: Methods of sedation in pediatric endoscopy: a survey of NASPGHAN members. J Pediatr Gastroenterol Nutr 2007; 45: 500-2.

5.- Daza W, Chávez E, Ceresa S, Pizarro B: Endoscopia digestiva alta en pacientes pediátricos. Rev Chil Ped 1997; 68: 20-3.

6.- Ruddloff T, Cabrera M, Biel F, Venegas G: Panedoscopia digestiva alta en el niño. Rev Chil Ped 1981,4: 289-94.

7.- $\quad$ Sury M, Smith J: Deep sedation and minimal anesthesia. Pediatric Anesthesia 2008; 18: 18-24.

8.- Finkel Y: Sedation and anestesia in pediatric endoscopy: one size does not fit all. J Pediatr Gastroenterol Nutr 2007; 44: 295-7.

9.- Tosun Z, Aksu R, Guler G, Esmaoglu A, Akin A, Aslan D, et al: Propofol-ketamine vs propofol-fentanyl for sedation during pediatric upper gastrointestinal endoscopy. Pediatric Anesthesia 2007; 17: 983-8.

10.- Kaddu R, Bhattacharya D, Metriyacool K, Thomas R, Tolia $V$ : Propofol compared with general anestesia for pediatric GI endoscopy: Is propofol better? Gastrointest Endosc 2002; 55: 27-32.

11.- Lightdale J, Valim C, Newburg A, Mahoney L, Zgieszwski S, Fox V: Efficiency of propofol versus midazolam and fentanyl sedation at pediatric teaching hospital: a prospective study. Gastrointest Endosc 2008; 3: 1-9.

12.- Thakkar K, El-Serag H, Mattek N, Gilger M: Complications of pediatric EGD: a 4 year experience in PEDSCORI. Gastrointest Endosc 2007; 2: 213-21.

13.- Malviya S, Voepel-Lewis T, Ludomirsky A, Marshall J, Tait A: Can we improve the assessment of discharge readiness? A comparative study of observational and objetive measures of depht of sedation in children. Anesthesiology 2004; 100: 218-24.

14.- Gana JC, Glenz C, Marchant P, Vaca C, García X, Larraín $F$, et al: Evaluación prospectiva de la seguridad y tolerancia de los procedimientos colonoscópicos en pacientes pediátricos. Rev Med Chile 2006; 134 : 613-22. 